\title{
A model of duplex structure for short vapour-grown carbon fibres
}

\author{
Antonio Madroñero, ${ }^{a}$ Edith Ariza ${ }^{a}$ and Marina Verdu ${ }^{b}$ \\ ${ }^{a}$ CENIM, Avda Gregorio del Amo 8, 28040 Madrid, Spain \\ ${ }^{b}$ CIDA, C/ Arturo Soria 289, 28033 Madrid, Spain
}

\begin{abstract}
Vapour-grown carbon fibres are a type of short carbon fibres produced by a single-stage process. They are not yet commercially available but their cost/performance ratio is excellent owing to their unusual inner microstructure. Nevertheless, much effort will be needed to achieve a fibre of this type with sufficient length and strength to be used as a composite reinforcer in the future. In this work a routine production is shown that yields fibres grown without any influence from the substrate. Under these conditions, their tensile behaviour and fracture are different from current ex-PAN fibres. A simple model is proposed for the evaluation of their strength by a measurement of the thickness of the two different layers that constitute each fibre.
\end{abstract}

Most $(90 \%)$ of the carbon fibres produced worldwide are exPAN and the remaining $10 \%$ are ex-Pitch. Both types are excellent reinforcers for composites, but their cost is so high that an increase in their consumption can hardly be expected. Nevertheless, the third-generation VGCF (vapour-grown carbon fibres) ${ }^{1}$ that until now had only been studied at laboratory level, are now appearing on the horizon. These are short fibres that are produced in a single stage using a gaseous hydrocarbon (methane, acetylene, benzene, etc.) as their raw material and employing a method similar to that used, for example, in the manufacture of the well known $\mathrm{SiC}$ whiskers, as described by Milewski et al. ${ }^{2}$

Aside from their moderate price (estimated for industrial scale production ${ }^{3}$ at some $\$ 5 / \mathrm{lb}$ ), their great advantage is that they are produced directly as short fibres. When present-day carbon fibres are used in normal manufacturing processes to produce high market-volume composite materials, such as injection moulding, sheet moulding compound (SMC) pro forma infiltration by resin transfer (RT), etc., ex-PAN or exPitch fibres must be chopped before use. This unavoidable chopping operation adds a practical and undesirable problem (blunting of cutting tools) which supposes an additional cost for the already expensive commercial carbon fibres. VGCF fibres are free from this problem.

The industrial future of VGCF depends mainly on their strength and length. In this paper we establish a very simple model to cover the wide range of strengths, from the point of view of their duplex structure and failure mode. This model allows an easy evaluation to be made of VGCF strength, as well as of the best choice of fibre thickness to achieve optimum strength.

\section{VGCF Production Processes}

It is widely accepted that the VGCF production process is a sui generis lampblack production from gaseous hydrocarbons. Basically, some minute particles of one of the transition metals $(\mathrm{Fe}, \mathrm{Ni}, \mathrm{Co})$, or an alloy of the same, are first placed in the reactor, so that the carbon reduced in the atmosphere is adsorbed by the metal particles that catalyse the decomposition reaction of the hydrocarbon. This adsorbed carbon is used to form carbides and, once the metal particles or seeds are saturated, the continuation of their catalytic effect means that the excess carbon is exhausted in the form of a cylindrical graphitic deposit. The fingerprint of these fibres is a 'treetrunk' structure, i.e. a fibre composed of successive concentric layers.

Briefly, the production technology for VGCF fibres permits two basic variations. In the process known as the aerogel method, ${ }^{4}$ tiny seeds are drawn along in a hydrocarbon and hydrogen atmosphere in such a way that when they reach the outlet collector, they have grown to a fibre of length $50-400 \mu \mathrm{m}$ with an aspect ratio (length/diameter) up to 100 . In the case of seeds lying on a steady bed, ${ }^{5}$ a solution of an $\mathrm{Fe}, \mathrm{Co}$ or $\mathrm{Ni}$ salt is applied with an atomizer to a strip or substrate. The production cycle consists of a first stage with hydrogen alone that converts the minute dried salty drops into active metal particles. Even with such a small range of dimensions the necessary surface/volume ratio can be achieved to reach a high level of catalytic activity. During the second stage of the process, a mixture of hydrocarbon and hydrogen (i.e. one typical composition is $30 \%$ methane and $70 \%$ hydrogen) causes fibre growth, forming a 'lawn' of short fibres that carpets the substrate.

It is generally agreed that the basic mechanism for the formation of $\mathrm{VGCF}^{6}$ is as indicated in Fig. 1. A metal particle adsorbs reduced carbon, depositing it on its lower end as graphite, so that the seed rises on the filament that is generated at its lower end.

Apart from the adsorption of catalysed carbon by seeds, there is a slight deposit of pyrolytic carbon that continuously covers the whole substrate and fibres, just as condensated water vapour from the atmosphere impregnates all the grass of a lawn with dew. When the thickness of this layer deposited on the substrate, on the body of the filament and on the tip where the rising and active seed is located, reaches a sufficient thickness to insulate the seed from the atmosphere from which it draws the carbon, the catalytic mechanism ceases. It is then said that the lengthening has terminated owing to poisoning of the seed. This is why VGCF fibres do not usually reach the length ${ }^{7}$ required by manufacturing processes for composite items using such processes as sheet moulding compound (SMC), body moulding compound (BMC), transfer resin moulding (TRM), etc.

If the situation following seed poisoning is excessively prolonged, all that occurs is that the fibres continue to thicken with the increasing amount of pyrolytic graphite coating their surface $^{8}$ If the crystallinity of this pyrolytic outer layer is not

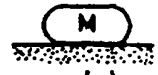

(a)

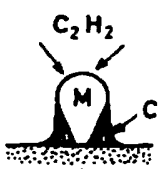

(b)

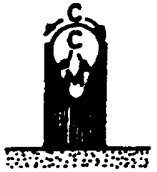

(c)

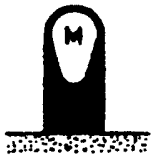

(d)
Fig. 1 Baker's model ${ }^{6}$ 
so good as that of the inner core, as Koyama ${ }^{9}$ established after electron diffraction studies, excessive thickening means a decrease in the tensile strength of a fibre that initially had a catalytic structure only. ${ }^{9}$

Thus, even though the production process for these fibres is rather simple, in order for VGCF to possess sufficient strength to make them desirable as industrial materials, the following points must be resolved.

(1) Although there are many theoretical models to explain the formation of VGCF, none of them explains, for example, the necessary requirement of hydrogen as the diluent gas. On the one hand, if we attempt to substitute hydrogen by another carrier gas such as helium or nitrogen, fibres do not form. On the other hand, according to the Mass Law, a reaction such as the decomposition of a hydrocarbon into carbon and hydrogen (hydrogen-generating reaction), would be easier under initial hydrogen-free conditions. This is totally opposite to the practice for producing VGCF, where the growth is carried out in an atmosphere containing $>50 \%$ hydrogen. It is impossible to consider a mature technology for VGCF without resolving this question. In this order of things, an understanding of the vapour-liquid-solid (VLS) process as described below could be very helpful.

(2) Optimization of the production process in order to achieve VGCF with mechanical properties competitive with present-day ex-PAN and ex-Pitch fibres is desirable. If the production process is continued until graphitizing occurs, the inclusion of graphite intercalation compounds is recommended, ${ }^{10,11}$ since this makes graphitization easier. In the particular case of current VGCF production (without intercalation compounds) there is another possibility for improving the mechanical properties in a way similar to graphitizing. If, immediately on termination of the manufacturing process, the produced VGCF are annealed in a pure hydrogen atmosphere at the process temperature, overhydrogenation of the fibres increases their adhesiveness and decreases their strength. After annealing for $45 \mathrm{~min}$, the Young's modulus of the thin VGCF is increased from 350 to $535 \mathrm{GPa}$, but their strength was diminished from 2620 to $1870 \mathrm{MPa}$.

(3) Improving the technique for rapidly and easily evaluating the mechanical characteristics of VGCF is desirable. Assuming that a tensile test of very short fibres requires equipment and practices as delicate as those in a study of the mechanical properties of whiskers, current tensile tests are too delicate to be used as a routine industrial control for evaluating the quality of each batch.

(4) It will be useful to achieve improvements to the production process, with particular reference to the average length of the filaments (which should not be less than a few $\mathrm{cm}$ long), according to the requirements of the manufacturing processes for items made of composite materials (SMC, RTM, etc.) mentioned above. Tsukamoto et al. ${ }^{12}$ state that it is possible to produce VGCF with lengths of up to $30 \mathrm{~cm}$, but they give no description of their process or of the reactor chamber.

\section{The Role of Hydrogen in the VGCF Growing Mechanism}

When a batch of VGCF is produced in a steady bed-type reactor, the fibre crop shows several filament morphologies (regular cylinders, curled, wormlike, etc.), indicating that small local variations (roughness of the substrate, seed size, local flow speed of reactant gases, etc.) have some influence on the growing mechanism. The basic VS model is that of Baker and co-workers, ${ }^{6,13}$ based upon the idea that the energy released by diffusion of hydrogen in the metal particles or seeds is the driving force for carbon filament formation (Fig. 1). The same direction is taken by Figuereido's study that establishes the starting of filament formation at $670{ }^{\circ} \mathrm{C}{ }^{14}$ In this type of VGCF their fingerprint is a hollow channel along the fibre axis.

A variation of the VS model is the process of carburetted seed, ${ }^{15}$ i.e. the reduced carbon produces oversaturation in carbon at the seed. Thus, an ordered carbon precipitation is produced toward the precipitation interface. The final result is the exhaust of a graphite filament, also with a hollow channel along the fibre axis.

The behaviour of the carburetted seed was modified by Gadelle et al..$^{5}$ and Baker et al. ${ }^{6}$ In this model the VS process is converted to VLS because the filament germination and growth starts with the carburetting of the seed, but after a short time it melts. The melting temperature depends on the seed size. For $6,10,15$ and $25 \mu \mathrm{m}$ particles, the melting temperature are $1020,1073,1100$ and $1121^{\circ} \mathrm{C}$, respectively, which are in the current temperature range for VGCF production. This VLS model does not presume the formation of a hollow channel at the centre of the fibre. Filament growth is more rapid because the diffusion of the products from the atmosphere now takes place in a liquid drop.

Looking for the influence of the metal constituent of the seed, we performed a very simple experiment, consisting of a VGCF without any metal seeds at all. Using equipment similar to that used by Benissad et al. ${ }^{5}$ we carried out a first stage consisting of the production of a coating of lampblack on the whole inner surface of the reactor; the working conditions were $70 \% \mathrm{H}_{2}+30 \% \mathrm{CH}_{4}$ as the reactant atmosphere, and $1060^{\circ} \mathrm{C}$. The second stage consisted of maintaining the same temperature for $1 \mathrm{~h}$, but in an atmosphere of $100 \% \mathrm{H}_{2}$. After that, the gas flow of $70 \% \mathrm{H}_{2}+30 \% \mathrm{CH}_{4}$ was restored. During the fourth cooling stage, only pure argon flowed. The result was that a certain proportion of carbon particles acted like the metal particles in the model of Benissad et al., ${ }^{5}$ adsorbing hydrogen, and regular VGCF were produced. Therefore, the model of Baker and co-workers ${ }^{6,13}$ was not accomplished because there was no hollow channel in the fibre centre. A model for the location of the stored hydrogen in the graphite network was reported, ${ }^{16,17}$ in which the accommodation of hydrogen $\mathrm{sp}^{2}$ and $\mathrm{sp}^{3}$ atoms in $\pi$-bonded clusters, was described.

The amount of collected fibres was scarce, but the filaments were very regular (not curled or branched). The composition of these fibres is undoubtedly different from the VGCF obtained from metallic seeds; Audier et al. ${ }^{18}$ performed X-ray diffraction tests, with the result that 'the diffraction spectra of the samples show diffraction lines of both the carbon and the metal. Whatever the experimental conditions are, no extra lines ascribable to a carbide have been observed'. In our fibres, no metal at all was present.

In an attempt to increase the number of fibres produced in a batch (fibres are more abundant when metal seeds are used), we prepared an operating routine as described below [see Fig. 2(a) ]. ${ }^{19}$ The basic aspect of this technique is to immobilize the metal seed by burying it in a shallow carbonaceous bed. Such a substrate has been proven to be productive (see above), and with this operating mode it is possible to prevent the ascent of the seed on the tip of the growing filament.

The first step was a precoating of the support or substrate with a carbonaceous coating produced by methane reduction $\left(950{ }^{\circ} \mathrm{C}, 50 \% \mathrm{CH}_{4}+50 \% \mathrm{H}_{2}, 10 \mathrm{~min}\right)$. The second was spraying with a solution of $\mathrm{Fe}\left(\mathrm{NO}_{3}\right)_{3} \cdot 9 \mathrm{H}_{2} \mathrm{O}$. Thirdly, the salt grains were reduced with $100 \% \mathrm{H}_{2}$ for $10 \mathrm{~min}$ at $950^{\circ} \mathrm{C}$. The temperature was then increased rapidly to $1075^{\circ} \mathrm{C}$, at which temperature the sample was held for $5 \mathrm{~min}$. The fourth step involved the deposition $\left(1075^{\circ} \mathrm{C}, 20 \% \mathrm{CH}_{4}+80 \% \mathrm{H}_{2}, 15 \mathrm{~min}\right)$ of a thin carbonaceous coating on the seed. Fifthly, $100 \% \mathrm{H}_{2}$ was passed over the sample for $45 \mathrm{~min}$ at $1075^{\circ} \mathrm{C}$. We think that during this critical stage, the seed surrounding was converted into hydrogen-oversaturated carbon. At the end of this stage, the atmosphere was changed to $70 \% \mathrm{H}_{2}+30 \% \mathrm{CH}_{4}$. The growing of VGCF then began. 
(a)

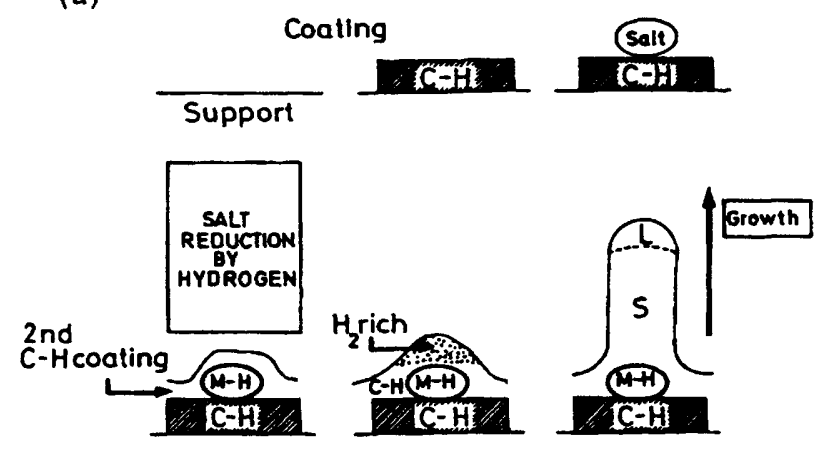

(b)

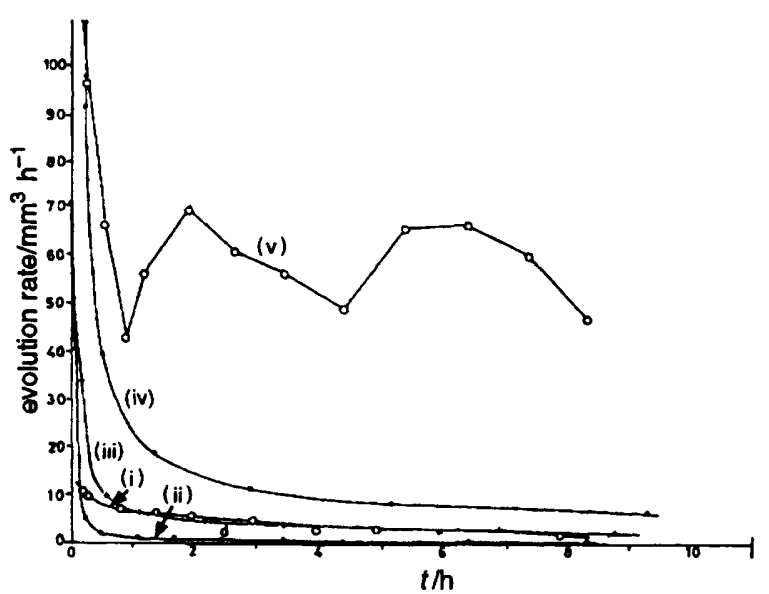

Fig. 2 VLS model for VGCF growth with Fe seeds. (a) Sequence of steps in the VLS process. ${ }^{19}$ (b) Evolution of the $\mathrm{H}_{2}$ content of $\mathrm{Fe}:^{25}$ (i) 25 , (ii) 440 , (iii) 675 , (iv) 925 , (v) $1075^{\circ} \mathrm{C}$.

It is reasonably simple to explain this VLS-type growing process in some detail. In Fig. 3(a), near the upper right-hand corner, it is possible to see how a fibre that is beginning to rise appears as a liquid droplet (contact angle $c a .90^{\circ}$ ), where the metallic seed lies shallowly buried. There is a clear resemblance between this sort of 'wart' and the initial rising of a silicon carbide whisker as shown in the work of Portnoi et al. ${ }^{20}$ Another, older fibre in the lower right-hand quarter of the picture, stops growing when its head touches an obstacle placed across its path from left to right. Its tip may be nonrigid because the integration of the tip in the obstacle appears to be quite close, which suggests a fibre tip with a good level of wettability. After SEM examination of many broken fibres of this type, no hollow channels were found. As the iron seed remains shallowly buried, the progression of the fibre tip during the growing process probably occurs with a liquid drop on the tip. After that, we could identify the composition of this melt tip.

We carried out another experiment to show clearly such a growth mechanism without any seed on the fibre tip. In Fig. $3(b)$ is shown a fibre which after production was treated in an $\mathrm{Ar}$ atmosphere $\left(\mathrm{ca} .1000^{\circ} \mathrm{C}\right)$ with a small proportion of water stream. Because of the reaction: water + graphite $=$ carbon monoxide + hydrogen, ${ }^{21}$ many regular pittings are produced. After this treatment, when the atmosphere is changed to pure hydrogen, these pittings behave as activated local areas where hydrogen is adsorbed.

If, after this treatment, the fibre shown in Fig. 3(c) is exposed to the proper conditions for the growing process $\left(1075^{\circ} \mathrm{C}\right.$, $70 \% \mathrm{H}_{2}+30 \% \mathrm{CH}_{4}$ ), the branching demonstrates how a VGCF can grow without any metal seed at all. The branches have the same tree-trunk internal grain as in all VGCF, as SEM examination of a cut branch shows. (a)

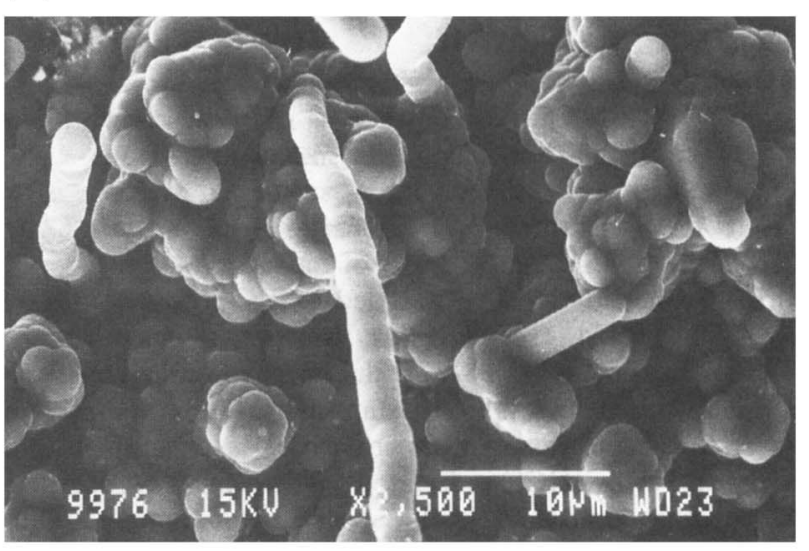

(b)

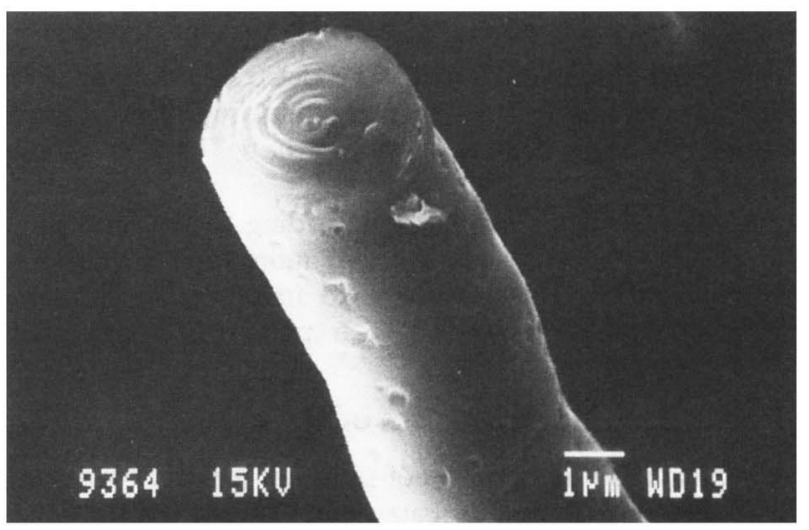

(c)

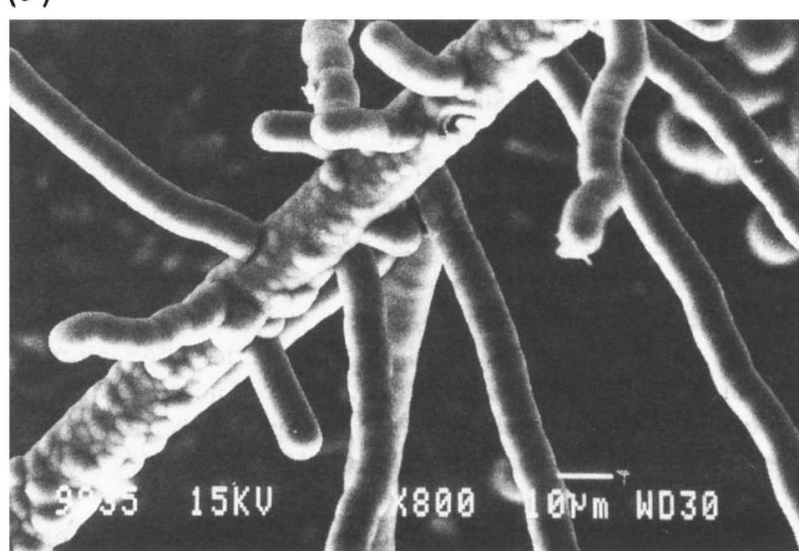

Fig. 3 Initial stages of fibre growth. (a) Initial growing stages; (b) pitting produced by $\mathrm{H}_{2} \mathrm{O}$ stream; (c) branching growth.

It is known that this branching can also take place in VGCF grown with an active carbide on the tip, ${ }^{22}$ but it requires carbide debris resting along the grown fibre, and in X-ray diffraction studies of our fibres no carbide line is identified.

In view of all these facts, we presume that the role of the metal seed, in the VLS model shown in Fig. 2(a), is to act as a hydrogen storer. All metals, to a greater or lesser extent, accept the entry of hydrogen, ${ }^{23}$ but group VIII elements are able to chemisorb hydrogen from the hydrocarbons so well that for many years they have been used as catalysts for hydrocarbon dehydrogenation. ${ }^{24} \mathrm{Fe}, \mathrm{Ni}, \mathrm{Co}$, etc., not only form carbides, but also store hydrogen in the known manner indicated in Fig. $2(b) .{ }^{25}$ This hydrogenation is not constant, and when the hydrogen is emitted at the proper moment, the surroundings of carbonaceous material are converted to compounds $\mathrm{C}_{n} \mathrm{H}_{m}$ at $1075^{\circ} \mathrm{C}$, as will be discussed here following a 
study performed by X-ray diffraction of VGCF. This study shows how the inner part of a VGCF is constituted of coronene $\left(\mathrm{C}_{24} \mathrm{H}_{12}\right)$.

Even though the pressure-temperature phase diagram is clear, ${ }^{26,27}$ that for the $\mathrm{C}-\mathrm{H}$ system is not well known and so it is not possible to calculate the size of melt drops, as was done for iron particles, ${ }^{8}$ but the experimental evidence is that VGCF are constituted by hydrocarbons of the type $\mathrm{C}_{24} \mathrm{H}_{12}$ (coronene), giving rise to drops of melted graphite (or, more correctly, melted $\mathrm{C}_{24} \mathrm{H}_{12}$ ) provided that the melting point of coronene $^{28}$ is $434-436^{\circ} \mathrm{C}$. In fact, we are not sure that during the growing process at $1075^{\circ} \mathrm{C}$ the fibre would be constituted of pure coronene; we can only state that at room temperature, when the X-ray measurements are made, the constituent of the inner part of a VGCF is $\mathrm{C}_{24} \mathrm{H}_{12}$. During the VGCF process the existing compound could be another compound $\mathrm{C}_{n} \mathrm{H}_{m}$, which is in thermodynamic equilibrium with $\mathrm{C}_{24} \mathrm{H}_{12}$ at $1075^{\circ} \mathrm{C}$. It is also possible that the variations in temperature, composition and properties might be continuous, without a real phase change. In this sense, the studies by Tamor and $\mathrm{Wu}^{29}$ on 'diamond-like' carbon describe the defected graphite model, which indicates the existence of a variable proportion of groups of stable hydrogen clusters as well as the $\mathrm{sp}^{3}$ - and $\mathrm{sp}^{2}$-bonded hydrogen.

The fibre-growth mechanism according to the VLS model then begins [Fig. 2(a)], and it occurs more rapidly than in the VS model with diffusion in the solid state (Fig. 1). What was previously referred to as catalytic graphite is in fact coronene.

\section{Mechanical Characteristics of the Fibres}

With the availability of fibres of sufficient length to allow easy manipulation, it is easy to carry out filament tensile tests in accordance with standard ASTM D3379-75, taking into account some procedural improvements described by Hagege and Bunsell. ${ }^{30}$ In accordance with this standard, the fibre under test is mounted onto a tab of Bristol paper, and this tab is then fixed to the tensile machine grips. After mounting the tab is cut so that only the fibre under test is connected between the two grips. The main question when performing such tensile tests, if good accuracy is required, is the technique used to evaluate the fibre cross-sectional area. In the ASTM standard an optical microscope with a Watson-type eyepiece is recommended. In our opinion, this is valid for fibres with a circular cross-section and a thickness greater than $6 \mu \mathrm{m}$; for thinner fibres the relative error is excessive. Therefore, commercial ex-PAN can be included in the range of fibres capable of being measured by optical microscopy, but thin VGCF cannot be tested in this way.

Our measurements of fibre thicknesses were made using the $\mathrm{He}-\mathrm{Ne}$ laser beam diffraction test; ${ }^{31}$ the fibre thickness is obtained using the Fraunhofer diffraction equation for a cylinder. Using this technique, when the fibre thickness is $c a .8 \mu \mathrm{m}$, the relative error in fibre diameter measurements is $c a .0 .5 \%$, but if the fibre thickness is $c a .5 \mu \mathrm{m}$, the error is $0.25 \%$. In contrast to microscopy, light diffraction is more accurate when used on thin fibres than when employed for thick ones. Thus, it is possible to measure fibre strengths with an error of $3 \%$. Working with these conditions for fibres thicker than $4 \mu \mathrm{m}$, the standard deviation in five samples was equal to or lower than the experimental error of $3 \%$. For fibres thinner than $4 \mu \mathrm{m}$ the standard deviation, owing to major irregularities in the fibres and the fibre handling difficulties, was very high; thus, the evaluation of these fibres is not valid. For tensile strength measurements we used a commercial gauge extensometer calibrator MTS $^{\circledR}$, in which an electronic load sensor was implemented; with this device it was possible to obtain stretchings as small as $2 \mu \mathrm{m}$.

Since the following results were obtained from VGCF fibres made in our laboratory following the VLS-like route described above, there may be some differences in comparison with published results corresponding to fibres grown in other laboratories via other processes.

As for carbon fibres produced from precursors other than those used to produce VGCF, many fracture features depend on the breaking mode of such samples and their internal grain and thickness (see Fig. 4). As there are important differences between the fractures of fibres broken under tensile conditions when the fibres are cut with scissors or with a lancet, all the fracture examinations of VGCF require the use of tensiled samples; in fact, sample fracture is the best way of exhibiting the microstructure of a VGCF. If the breaking occurs under tension, in thin fibres with only catalytic graphite, the break is as fragile as commonly appears in ceramic fibres. In thick fibres with a thick pyrolytic graphite coating, the break causes the shear cracking of the pyrolytic/catalytic graphite interphase, giving rise to a 'sheath-and-sword' failure. ${ }^{32}$ In the SEM examination of the fracture it is advisable for half of a broken fibre to be examined [see Fig. 5(a)]. If some feature is observed that suggests an imperfect tensile failure (preexisting damage or defect), the result of such a tensile test must not be taken in account

Turning to the interpretation of the results of VGCF tensile tests, VGCF can be divided into three mechanical ranges [see Fig. 5(b)], according to the relative content of catalytic/pyrolytic grains included in the duplex structure. These three types are: very thin, medium and thick, and they should have three different strength-thickness relationships.

The medium-thickness fibres should maintain a nearly constant value of tensile strength, $\sigma$, corresponding to the 'treetrunk' structure. The very thin ones should have an ascending function $\sigma-\phi$ ( $\phi=$ fibre thickness; because such a function contains the $\sigma=0$ and $\phi=0$ point), as the beads that form the fibre become more continuous and the fibre takes on the aspect of a perfect cylinder with no significantly narrower sections. Thick fibres should lose their strength as their pyrolytic carbon coating becomes thicker, in proportion to the amount of pyrolytic graphite/catalytic carbon contained. Obviously the pyrolytic carbon is more vitreous and weaker [see Fig. $4(d)]$ than the catalytic one.

Applying the rule for mixtures, the tensile strength $(\sigma)$ of a thick fibre will be:

$$
\sigma=\sigma_{1} \frac{\pi r^{2}}{\pi R^{2}}+\sigma_{2} \frac{\pi\left(R^{2}-r^{2}\right)}{\pi R^{2}}=\left(\frac{r}{R}\right)^{2}\left(\sigma_{1}-\sigma_{2}\right)+\sigma_{2}
$$

where $r$ is the tree-trunk inner core radius, and $R$ is the outer radius, as shown in Fig. 5(a). Eqn. (1) means that the value of $\sigma_{1}$ will be identifiable with the value of $\sigma$ for optimal fibres (very thin pyrolytic coating, $[R-r] \approx 0$, i.e. $R \approx r$, and so $[R / r] \approx 1)$, without the narrower necks that exist in very thin fibres. On the other hand, for very high values of $R(R \gg r$, such that $r / R$ is negligible), the strengths of very thick fibres will be identifiable with $\sigma_{2}$.

We shall attempt to statistically adjust the experimental data of Fig. 5(a), which may be expected to be the optimum for quadratic second-degree functions, given eqn. (1). Rather than adjusting the whole range of experimental data with only one mathematical expression, it would be better to ignore the data for very thin fibres, and to adjust only the data for the medium thickness and very thick fibres with different mathematical expressions. In this way, for medium thickness fibres we obtained [ $\sigma$ in $\mathrm{MPa}$ and fibre thickness $(\phi)$ in $\mu \mathrm{m}]$ :

$$
\sigma=2526+46 \phi-4.7 \phi^{2}(c=0.95, \text { regression coefficient })
$$

and for thick fibres, we found:

$$
\sigma=4200-126 \phi-0.4 \phi^{2}(c=0.91)
$$

Using eqn. (2) we calculate the maximum value of $\sigma$ to be 
(a)

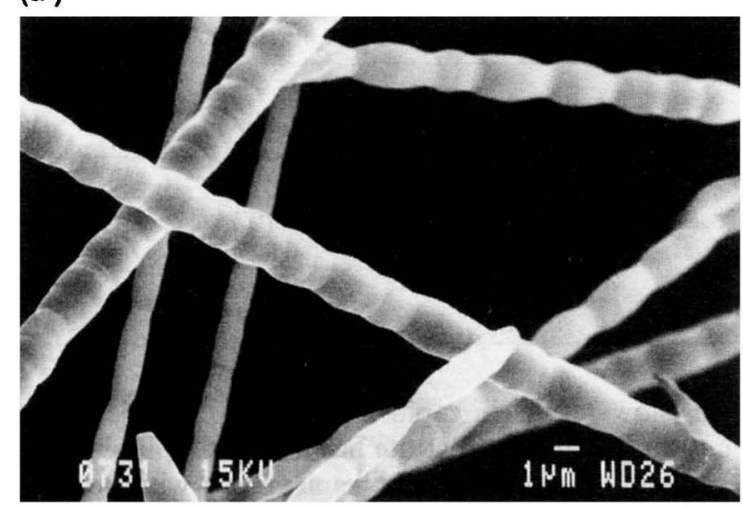

(c)

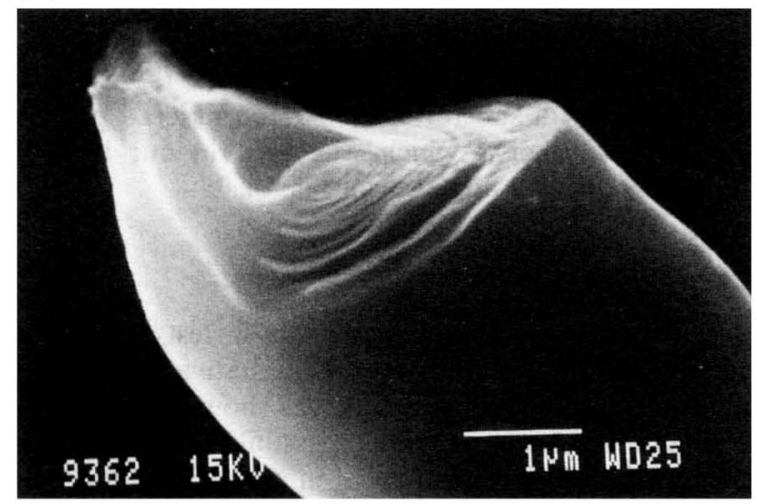

(b)

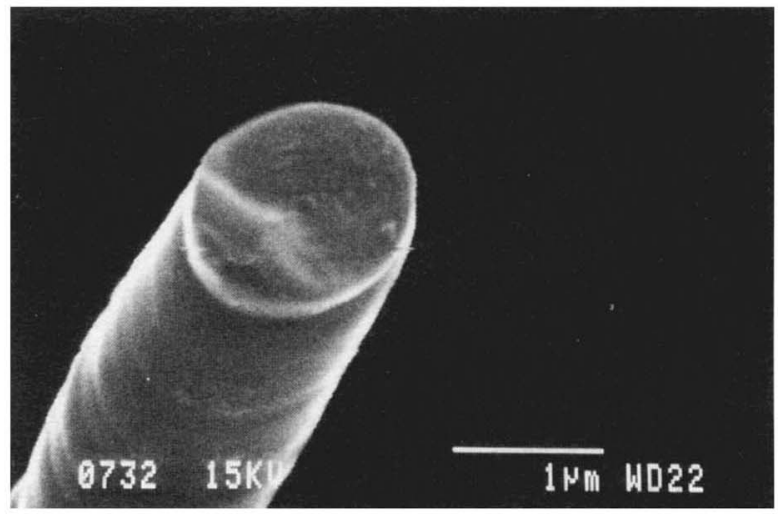

(d)

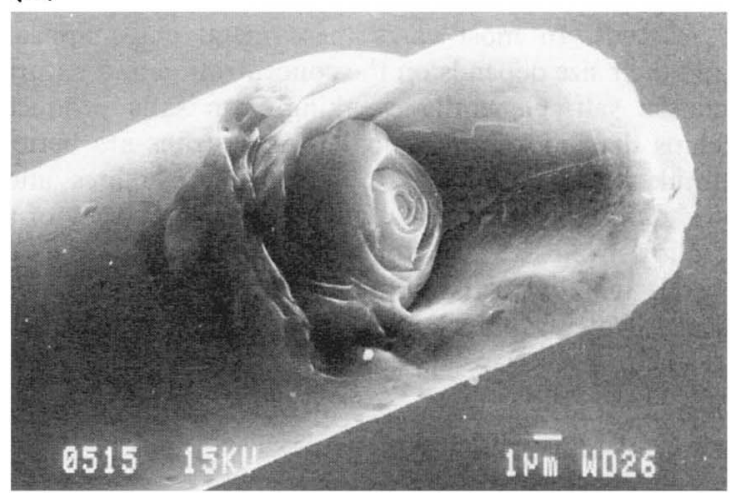

Fig. 4 Relationship between VGCF thickness and tensile fracture. (a) Very thin fibres; $(b)$ tensile fracture of very thın fibres; $(c)$ tensile fracture of medium thickness fibres, $(d)$ thick fibres

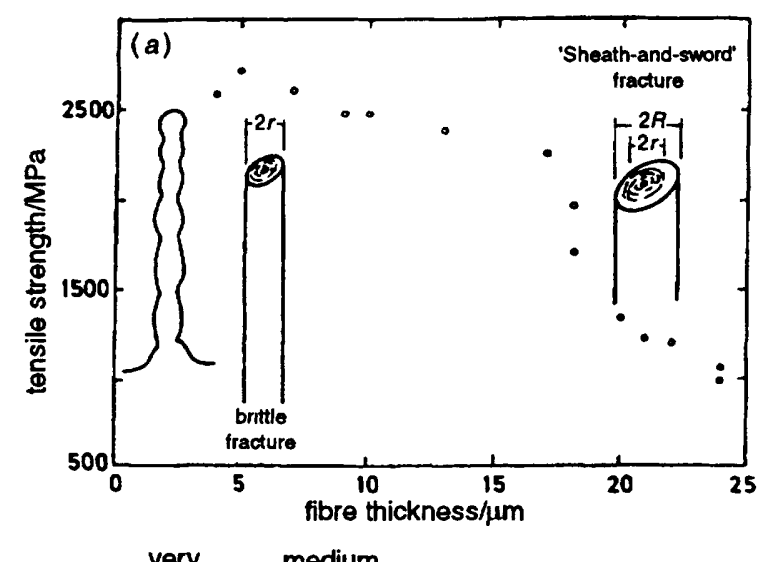

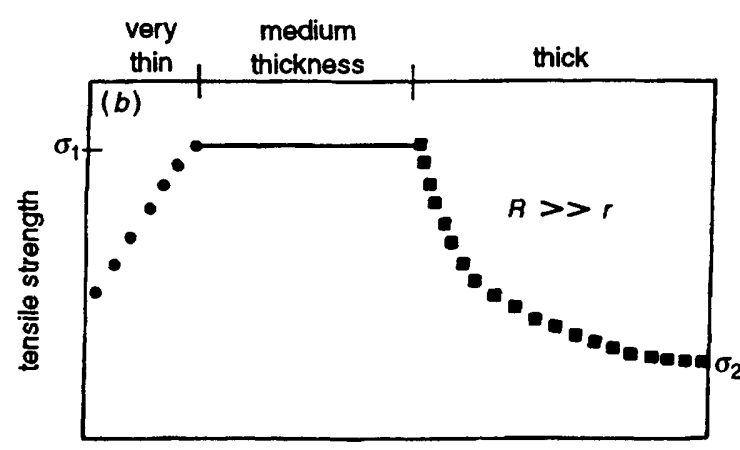

fibre thickness, $R$

Fig. 5 Relationship between tensile fracture and strength (a) Experimental measurements; (b) physical model $\sigma_{\max }=2638 \mathrm{MPa}$, which corresponds to $\phi_{\text {optimal }}=4.89 \mu \mathrm{m}$. This $\sigma_{\max }$ value can be identified as $\sigma_{1}$ from eqn. (1).

Eqn. (3) indicates that at $\phi \approx 30.4 \mu \mathrm{m}$ the value of $\sigma$ must be almost zero. In practice, this thickness of $30 \mu \mathrm{m}$ cannot be achieved. The thickest obtainable fibre is $c a .20 \mu \mathrm{m}$, which gives, from eqn. (3), $\sigma_{\text {min }}=1520 \mathrm{MPa}$; this value can be identified as $\sigma_{2}$ from eqn. (1)

In summary, eqn. (1) offers an easy and quick tool for a rough evaluation of the strength of VGCF or of the degree of optimization of the experimental conditions during the fibre manufacturing process. For example, if a batch of very short VGCF is obtained, the mechanical evaluation of these fibres by tensile tests in accordance with standard D3379 establishes is impossible. However, an alternative, rough evaluation can be performed a simple SEM examination of the transverse cross-section of tensile broken fibres, which enables the evaluation of the ratio $r / R$. The substitution of this value in eqn. (1), together with $\sigma_{1}=2638 \mathrm{MPa}$ and $\sigma_{2}=1520 \mathrm{MPa}$, yields an excellent routine strength approximation for the VGCF. An example of the application of this evaluation through the ratio $r / R$ is given in Table 1 (later)

An additional use of these values is in attempting to produce batches of VGCF samples in which most of the fibres possess the optimum strength. It is possible to select the best experimental conditions for the synthesis of optimum-strength fibres on the basis of the rough evaluation $\left(r / R, \sigma_{1}\right.$ and $\left.\sigma_{2}\right)$ described above of the fibres produced in each batch.

Another interesting topic is the influence of the seeds on the quality of the produced fibres. As shown in Fig. 2(a), the thickness of the tree-trunk structure of a VGCF is, to some extent, proportional to the size of the seed. Therefore, by controlling the dimensions of the seeds (equivalent to choosing a proper metallic salt solution and its concentration), and by 
Table 1 Comparison between diffraction and SEM approximate evaluations and tensile measurements

\begin{tabular}{|c|c|c|c|c|c|}
\hline \multirow[b]{2}{*}{ diffractogram of VGCF, Fig. 8} & \multicolumn{2}{|c|}{ evaluation from SEM and eqn. (1) } & \multicolumn{3}{|c|}{ tensile test measurements } \\
\hline & $r / R$ & $\sigma / \mathrm{MPa}$ & $\begin{array}{c}\text { fibre } \\
\text { diameter } / \mu \mathrm{m}\end{array}$ & breakıng load/N & $\sigma / \mathrm{MPa}$ \\
\hline (a) & 0.93 & 2486 & 4.53 & 0.056 & 3500 \\
\hline (b) & 0.90 & 2425 & 7.57 & 0.107 & 2390 \\
\hline (c) & 0.68 & 2036 & 5.32 & 0.047 & 2150 \\
\hline (d) & 0.55 & 1858 & 8.80 & 0.097 & 1610 \\
\hline (e) & 0.40 & 1698 & 6.50 & 0.053 & 1620 \\
\hline
\end{tabular}

using a minimal process time to produce a pyrolytic outer layer that is as thin as possible [beginning of the plateau in Fig. 5(b)], the optimal VGCF can be produced. The best method of controlling the seed size, is to make an appropriate choice of salt concentration. In Fig. 6, two metal particles are shown (salt grain later to be reduced) which are prepared to be used as seeds. In most cases these metal chips appear spongy and their size depends on the concentration and nature of the chosen salt. Generally speaking, iron salts produce smaller chips than nickel and cobalt salts; nitrites are better than chlorides, and chlorides are better than sulfates and carbonates. Our experience has shown that the efficiency of a metal or alloy, from the point of view of its behaviour as a seed, depends mainly on its ability to adsorb hydrogen. We obtained excellent results using $\mathrm{Ti}_{2} \mathrm{Fe}$, owing to its excellent ability to adsorb hydrogen. ${ }^{33}$

\section{Production of Longer Fibres}

It is known that the length of the VGCF produced depends on the flow speed of the reactant gas mixture in the reactor; a

(a)

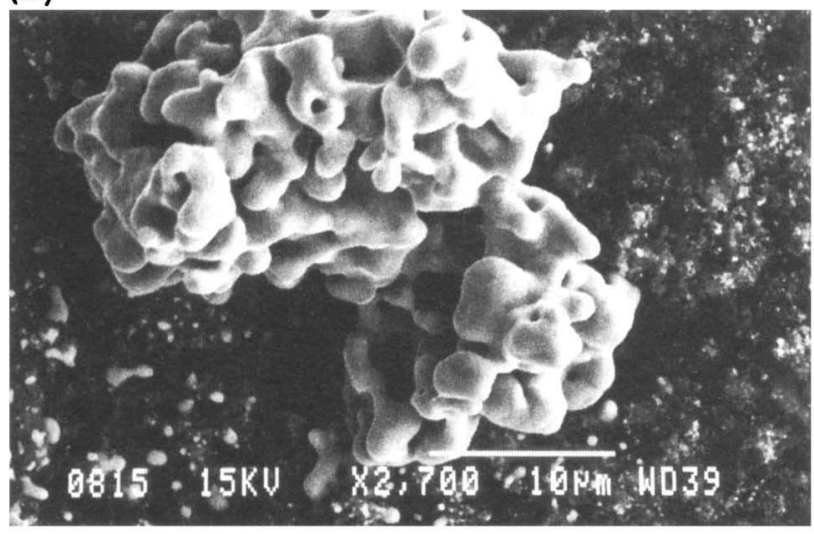

(b)

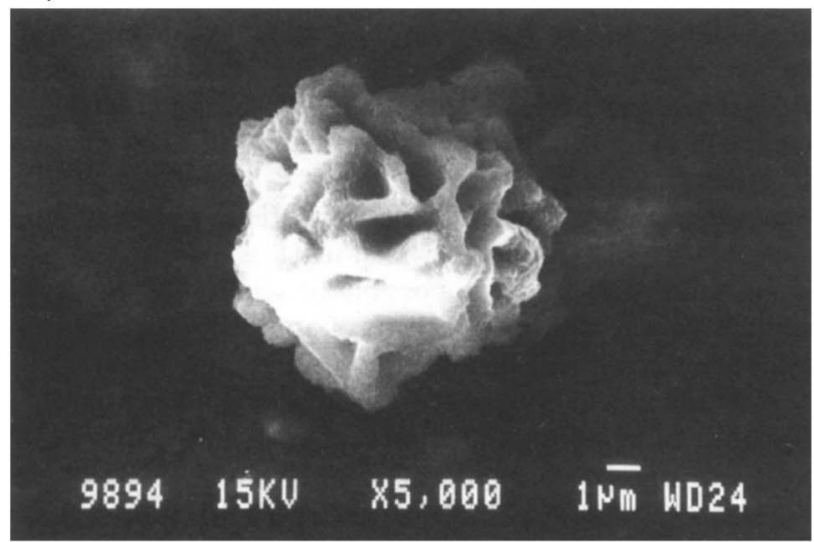

Fig. 6 Samples of metal chips produced by the reduction of droplets of the metal salt solution in hydrogen. (a) Large seed; (b) small seed. rapid gas flow results in rapid fibre growth. ${ }^{34}$ It is also very frequent that when the substrate has a very simple shape, such as a cylinder, ${ }^{35}$ the produced fibres are short with no relation to the seed aspect, shape and composition. In our opinion, with this very simple substrate geometry the gas flow forms a stagnant gaseous layer just on the substrate surface, in which the seeds are almost stifled. Therefore, a good solution to this problem is to keep the seeds in the middle of the gas stream. In Fig. 7 we show a substrate, similar to one used previously to grow $\mathrm{SiC}$ whiskers, ${ }^{2}$ which was used in our laboratory produced VGCF with an excellent length.

\section{X-Ray Diffraction of Carbon Fibres}

Because the performance of many tensile tests of VGCF is tedious, it may be interesting to find alternative approximation techniques for their evaluation. As was shown earlier, SEM is a useful tool, but there are other possibilities. Otherwise, the characterization of fibres produced in a batch is not easy.

One alternative technique is of X-ray diffraction, which can be used to characterize a bundle of fibres, as representative of a whole batch. Carbon fibre X-ray diffraction tests can be carried out in a Debye chamber of diameter $57 \mathrm{~mm}$. From the results obtained it is possible to see how the general features of the X-ray diffractogram are a good match for the average strength of the fibres. There are extensive collections of commercial diffractograms for ex-PAN fibres, ${ }^{36}$ in which the different grades of graphitization are correlated with some features of the diffractograms, which are strongly affected by the texture of the samples. ${ }^{37}$ However, the relationship between the diffractogram and the fibre strength is not so simple because the fibre strength is also affected by other aspects. Failure mechanisms in ex-PAN fibres include flaw displacement, the role of impurities, etc. As well as this, simple relationships between the level of graphitization and the strength of ex-PAN fibres are not easily transferable to VGCF, because ex-PAN fibres have a hexagonal crystalline structure, whereas VGCF are monoclinic.

From the practical point of view, the best method of resolving these problems would be to record the diffractograms of a whole range of VGCF previously evaluated by other techniques. By changing the seed size and the process time we can obtain a wide range of fibres with different $r / R$ ratios. Owing to the different degree of crystallinity in the core and outer materials that constitute the duplex structure of VGCF, we can see in Fig. 8 how the $r / R$ ratio determines changes in the

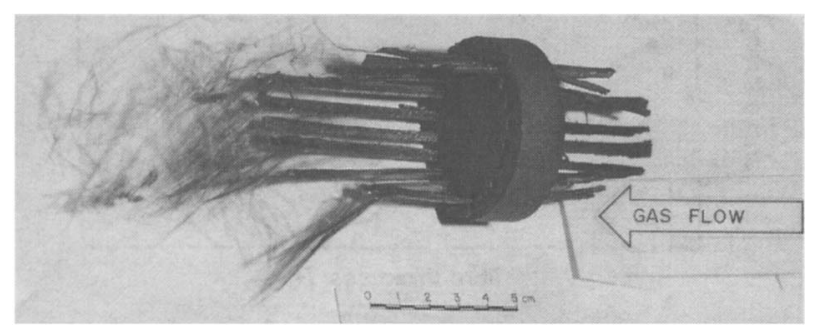

Fig. 7 Substrate used for VGCF growth 
shapes of the (104) and (102) rings. With ex-PAN fibres these diffractograms would be a little different because the texture is shown in the (002) and (004) planes. ${ }^{37}$

More strictly speaking, the diffractogram of VGCF is a mixture of two sets of diffraction lines, a set related to a hexagonal grain that corresponds to the pyrolytic phase, and another which reflects a monoclinic structure, corresponding to the catalytic phase.

A rough interpretation of VGCF diffractograms is sometimes rather simple. For example, it is very easy to confuse the strongest lines of both sets, (104) and (102) with (002) and $(004)$, but when a line such as $(110)$ from the hexagonal set is clearly visible as in Fig. $8(a)$, we know that we have a fibre with a thick outer coating.

In Table 1 the results are shown of the measurements of fibre strength by a tensile test, together with the evaluation of fibres of the same bundle (grown identically as a whole and at the same time in the reactor) via SEM examination. The diffractograms of Fig. 8 were taken from the spare fibres of each bundle.

A diffraction test can be an excellent tool to evaluate the level of strength of a produced batch of VGCF, by means of calibration with the samples of Fig. 8.

\section{Conclusions}

VGCF fibres manufactured using the VLS-like process, as described in this work, offer a very interesting alternative to the commonly used carbon fibres, in terms of cost and product size. By careful choice of both the salt solution to produce the seeds and of the growing conditions in the furnace, fibres can be obtained with an acceptable mechanical strength. An approximate estimation of VGCF strength can be obtained simply by using a related measurement for the core fibre diameter and the thickness of the pyrolytic outer layer. As distinct from ex-PAN fibres, and in a more semi-quantitative manner, an estimate of the tensile strength of VGCF can be made using a very simple test such as X-ray diffraction in a Debye chamber.

\section{References}

1 G. G. T1bbetts, Carbon, 1989, $27,745$.

2 J. V. Milewskı, F. D. Gac, J. J. Petrovic and S. R. Skaggs, J. Mater. Scl., 1985, 20, 1160.

3 S. Beck, How to apply advanced composites technology, ASM Internatıonal, Dearborn, Michigan, 1988, p. 348.

4 M. Endo, Chemtech, 1988, 568.

5 F. Benıssad, P. Gadelle, M. Coulon and L. Bonnetain, Carbon, $1988,26,61$.

6 R. T. K. Baker, M. A. Barber, F. S. Feates, P. S. Harris and R. J White, J. Catal., 1972, 26, 51.

7 G. G. T1bbetts, J. Cryst. Growth, 1985, 73, 431.

8 P. Gadelle, Carbon Fibres, Filaments and Composites, Kluwer, Dordrecht, 1990, p. 95.

9 T. Koyama, Carbon, 1972, 10, 757.

10 J. Shioya, H. Matsubara and S. Murukamı, Synth. Met., 1986, 14, 113.

11 J. S. Speck, M. Endo and M. S. Dresselhaus, J. Cryst. Growth, $1989,94,834$.

12 J. Tsukamoto, K. Matsumura, T. Takahash 1 and K. Sakoda, Synth. Met , 1986, 13, 255.

13 R. T. K. Baker, P. S. Harrıs, R. B. Thomas and R. J. Waite, J. Catal., 1973, 30, 86.

14 J. L Figueiredo, Wiss. Tech., 1989, 42, 294.

15 G. G. Tibbetts, in Carbon Fibres, Filaments and Composites, ed. J. L. Figuereido, NATO ASI Series, Kluwer, Dordrecht, 1990, p. 525 (a)

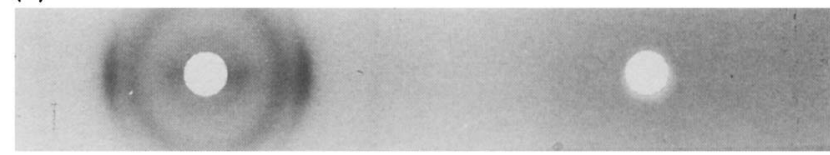

(b)

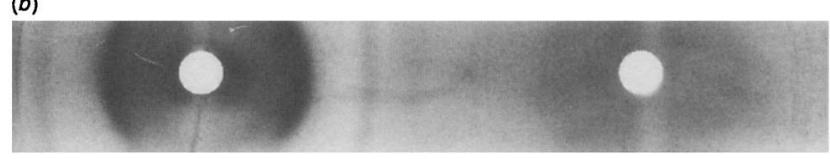

(c)

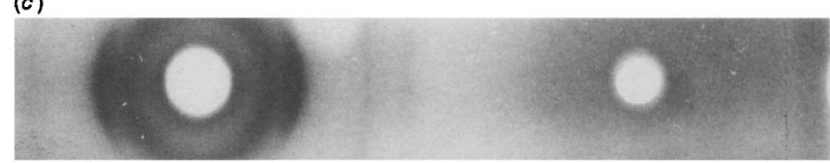

(d)

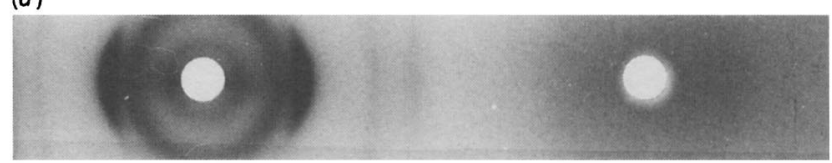

$(\theta)$

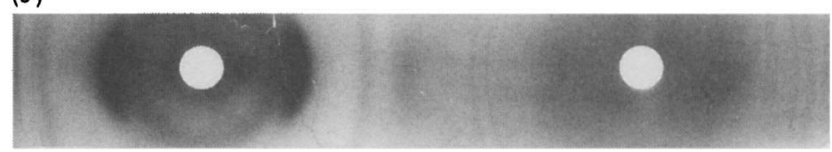

Fig. 8 Debye diffractograms of five VGCF samples (Cr-K $\alpha$ radiation). (a) $r / R \approx 0.93 ;(b) r / R \approx 0.90 ;(c) r / R \approx 0.68 ;(d) r / R \approx 0.55 ;(e) r / R \approx 0.40$.

16 J. Robertson, Adv. Phys., 1986, 35, 317

17 J. Robertson, Philos. Mag. B, 1992, 66, 199

18 M. Audier, J. Guinot, M. Coulon and L. Bonnetain, Carbon, 1981, $19,99$.

19 A. Madroñero, J. Mater. Scl., 1994, 30, 2061.

20 K. I. Portno1, A. A. Mukaseev, V. N. Gribkov, A. S. Isa1kın and E. L. Umantev, Sov. Phys. Crystallogr. (Engl. Transl.), 1974, 19, 198.

21 J. D. Blackwood and F. McGrory, Aust. J. Chem., 1958, 12, 16.

22 A. Oberlın, M. Endo and T Koyama, J. Cryst. Growth, 1976, 32, 335.

23 Y. Fuka1, The Metal-Hydrogen System, Springer-Verlag, Heidelberg, 1993.

24 Y. Tamai, Y. Nishiyama and T. Tateyama, J. Cryst. Growth, 1969, 14, 394.

25 D. P. Smith, Hydrogen in Metals, The University of Chicago Press, 1948 , p. 55.

26 F. P. Bundy, Physica A, 1989, 156, 169

27 K. J. Hüttınger, Adv. Mater., 1990, 2, 349.

28 J. Murdoch and T. A. Geisman, Am. Mineral., 1967, 52, 611.

29 M. A. Tamor and C. H. Wu, J. Appl. Phys., 1990, 67, 1007.

30 R. Hagege and V. Bunsell, in Fibre reinforcements for composite materials, ed. V. Bunsell, Elsevier, Amsterdam, 1988, p. 479.

31 L. J. Lynch and N. Thomas, Text. Res. J., 1971, 41, 568.

32 G. G. T1bbetts, M. Endo and C. P. Beetz, SAMPE J., 1986, 30.

33 J. J. Reilly and R. H. Wiswall, Ind. Chem., 1974, 13, 218.

34 T. Masuda, S. R. Mukaı and K. Hashimoto, Carbon, 1992, 30, 124.

35 G. G. Tibbetts, in Carbon Fibres, Flaments and Composites, ed. J. L. Figuereido, NATO ASI Series, Kluwer, Dordrecht, 1990, p. 73.

36 H. A. Goldberg, Final report to the US Army Research Office, Contract No. DAAE29-81-C-0016. A brief reference can be found 1n: M. S. Dresselhauss, G. Dresselhauss, K. Sugihara, I. L. Spain and H. A. Goldberg, Graphite Fibres and Fllaments, Springer Verlag, Berlın, 1988, p. 53.

37 D. J. Johnson, in Carbon Fibres, Flaments and Composttes, ed J. L. Figuereido, NATO ASI Series, Kluwer, Dordrecht, 1990, p. 128.

Paper 6/00888G; Received 6th February, 1996 